\title{
Channel wave tomographic imaging method and its application in detection of collapse column in coal
}

\author{
Wei Wang ${ }^{1}$, Xing Gao ${ }^{4}$ \\ Institute of Geographic Sciences and \\ Natural Resources Research \\ Chinese Academy of Science \\ Beijing, China
}

\author{
Guoqiang $\mathrm{Xue}^{2}$, Jiwen Teng ${ }^{5}$ \\ Institute of Geology \& Geophysics \\ Chinese Academy of Science \\ Beijing, China
}

\author{
Huanyu Cui ${ }^{3}$ \\ Hebei Coal Research Institute \\ Xingtai, China
}

\begin{abstract}
Coal seam seismic survey collapse column is common in heterogeneous geologic bodies. Groud-3D seismic observation due to limitations of its accuracy, for uneven geological subsided column often small leakage test or position error is large, the impact of mining coal mine safety sometimes causing heavy casualties. Downhole observation of channel wave transmission is one of the most effective ways to detect the small anomalous bodies within mine working face. Transmission experiments carried on at the king coal mine working face, in SHANGXI province, with 56 excitation points at lane and 48 receiving points at next lane arranged, its interval distance is $150 \mathrm{~m}$ along roadway. 1344 airy phrase travel time are picked through the dispersion analysis and tomographic inversion calculation of velocity and absorption coefficient is carried out. The distribution diagram of group velocity and absorption coefficient of the channel wave is built which obtain 10 anomalous bodies discovered and excluding the other collapse columns delineated by the three-dimensional seismic exploration in the test areas. There are number 1-9 collapse columns among the 10 anomalous bodies, Number 1-9 collapse columns are verified by the result of actual mining, and the location all deviation is less than $10 \mathrm{~m}$. Number 1 , 6and 7 collapse columns are exposed by the roadways. Number 6 collapse column has a $40 \mathrm{~m}$ 's extending length into the mining face. Number 7 collapse column has a $20 \mathrm{~m}$ 's extending length into the mining face. Number 4 collapse column has 50m's major axis and $30 \mathrm{~m}$ 's minor axis. This is consistent with the location of the collapse column DX4 given by 3D seismic exploration. There is no abnormal of the collapse column DX5 and DX6 in the result of tomographic inversion, which is exposed by 3D seismic exploration. The existence of Number 2-5 collapse columns seriously influences the coal mining safety. Concerning the geological condition exposed by the roadways, the production scheme has been modified to ensure high production and high productivity and safety of mine.
\end{abstract}

Keywords-collapse column, channel wave, dispersion analysis, tomographic imaging.

\section{INTRODUCTION}

Most coal mine accident is caused by abnormal feature geological structure body not detected in the coalfields, in which collapse columns as a key geological reason affecting the safe production ${ }^{[1]}$. Due to cavity, which result by the underground water years activities corrosion of around soluble rocks in the lower part of the coal-bearing stratum, the upper coal-bearing rock strata sink that formed the collapse column at the action of gravity. Usually collapse column is evolution from all upper disarray fall consist of uneven block cemented and piled and accumulate. $\mathrm{t}$ the hydrogeological complex conditions regions, subsided column as groundwater channel, the flooding accident occur when drilled through during coal mining process, raising huge economic losses and threatening the safety of mine workers.

Conventional two-dimensional, three-dimensional reflection seismic exploration method in coal mine exploration run well on ground, however at the scope of focused on collapse column prospect results reached, it exist heavy deviation location to actual from coal seam section explanation for less accurate on ground, usually the rang of collapse column area are far large to actual, it even not detect out which diameter is smaller. It is getting more dangerous along with continuous mining because of accumulation of small breaks, collapse columns and the hydraulic enclaves, this issue is getting worse and worse.

Contrast to the surrounding rock, the strata continuity, lithological characters, continuity of and so on , disparity leading to the normal reflection wave group not continuous or energy getting weak ${ }^{[2]}$. In collapse column simulation and identification, generally based on ray theory by diffraction waves of collapse column rift point, unfortunately the reflected waves hard observed outside the rift point and cylindrical case steep ${ }^{[4]}$. Even though significant change in lithology between collapse column and rock, wave impedance obvious huge distinction, subsided column generally as absorb medium, so the wave reflection energy turn more weaker after through it interface. In actual production, geometric boundaries difficultly clear imaged in the migration profile ${ }^{[5]}$ since the complexity in the spatial distribution and the particularity of seismic response. The interpretation of collapse column mainly be based on data of seismic reflected waves, together with coherent technology, take use of coal seam continuity or the feature reflected wave of its to forecast. Therefore, conventional two-dimensional, the three-dimensional reflection seismic exploration do high deviation to delineate actual position of the collapse columns, furthermore to the extent the area rang greater than the actual ${ }^{[6]}$. 
A new approach for accurate prospect column generated with rapid development of the channel wave seismic exploration technology in recent years ${ }^{[7]}$.In 2001, the United States launched the "Detecting Gob geophysical feasibility plan" includes a four slot-wave prospect research project ${ }^{[8]}$, promote greater development of channel wave exploration. 3D simulation calculate of channel waves in recent years become a hot topic with parallel computer development: the seismic waves excited on ground surface how to convert channel waves, and relations dependence on medium parameters [9] channel wave feature with the roadway, without roadway ${ }^{[10]}$ channel wave high-order phase characteristic etc.

Channel wave tomography in resolving faults, coal seam thickness variation get new progress, the study experiment aim to , the first is geological factors determines such as collapse column shape size, location, nature character insensitive to stopping progress smoothly; the second is the minimum resolution that fall wave through Column, whether collapse column excite scattering channel wave and coal seam roof or floor discontinuities cause the forward scattering. 9\# Wang coal mine, in Shouyang paragraph Shanxi province, as carboniferous system Taiyuan group, collapse column densely distributing in the working face, is idea experimental field for channel wave collapse column, in September 2011 at 090606 Face transmission experimental work conducted. The prospect result is consistent with the verification, all this lower mining costs, improve the production efficiency.

\section{THEORY AND METHOD}

\section{A. Channel Wave Transmission Imaging Principle}

Channel wave transmission tomography technology usually based on typically utilize different phase or frequency speed / travel time information tomography, can also use different types of transmission factor /attenuation coefficient imaging, such as amplitude ratio of the high and low frequency, relative transmission coefficient of high and low velocity group energy and Eric phase envelope attenuation coefficient imaging.

The principle of Channel wave tomography based on travel time is divided into different speeds grid along the ray path, the ratio of each grid travel distance and speed addition equal the total travel time of this wave ray; channel wave tomography based on amplitude attenuation is divided different attenuation coefficient grid according each ray path, that the product of each grid path and attenuation coefficient within the grid path add together, equal to the sum of each ray amplitude attenuation. The two way no distinction, solving multivariate linear equation group attenuation coefficient In mathematical, all can achieve by the conjugate gradient method (CG), least squares method (LSQR), algebraic reconstruction Technique (ART), the instantaneous iterative method (SIRT) etc algorithms.

This paper describes the amplitude attenuation coefficient method. Seismic wave propagation in the process, because of the absorption of the medium to seismic wave's energy, according to propagation distance amplitude attenuation, seismic wave energy received can be used (1) the expression:

$$
A(r)=A_{0} e^{-\alpha r}
$$

is the initial energy, $\alpha$ is the attenuation coefficient, $r$ distance from source point to receive point, $A(r)$ energy received,

The equation (1) is deformed (2):

$$
\ln (A(r))=\ln \left(A_{0}\right)-\alpha r
$$

Assume on the one side roadway explosives excited as source waves, the other side roadway to received, obtain a plurality data, the channel wave of the same frequency, if coal seam media uniform, by the equation (2) elucidate that the relation of the amplitude logarithmic and distance received is a linear function, elucidated in Figure 1, received amplitude logarithmic marked on the map with distance, change at linear law. Select all obvious data of groove wave received in a gun set, conduct regression analysis to get the initial amplitude and theoretical attenuation coefficient, and then find each channel amplitude attenuation coefficient according to the theoretical and actual amplitude difference.

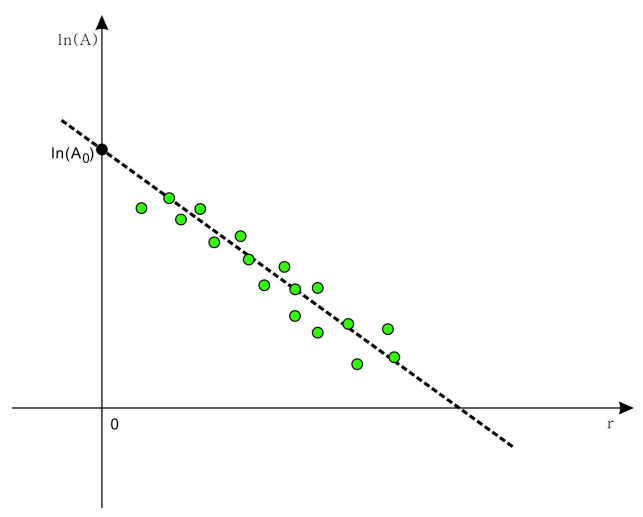

Fig. 1 Channel wave initial amplitude and absorption coefficient diagram

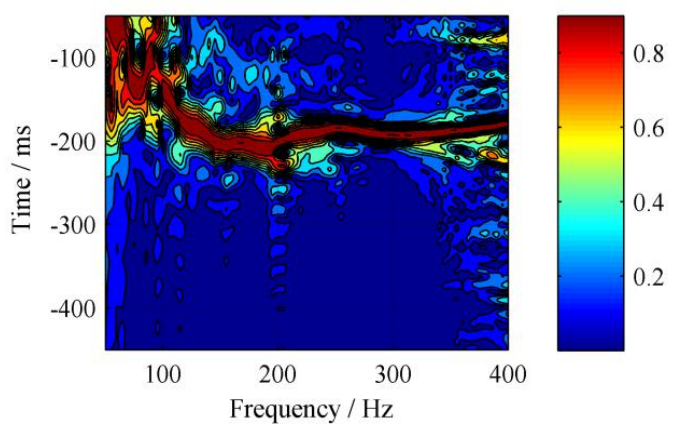

Fig.2 Inside area Shot No.27 and receiver No.16 dispersion analysis

$$
\ln (A)_{k}^{\text {calc }}=\sum_{j=1}^{N}\left[\left(\ln \left(A_{0}\right)-\alpha_{j} r_{j}\right]\right.
$$

The $\mathrm{k}$ rays path relationships with absorption coefficient on the grid are composition equations according to the formula (3) 
calculation, solving the equations to obtain the absorption coefficient distribution within the grid. In the case of coal seam stable, airy phases frequency and energy all elated to absorption coefficient. This method also be completed at common detection point domain after the amplitude consistency correction. By dispersion analysis (Fig.2) obtain different frequencies travel time of channel wave received, and calculate the amplitude of the received channel energy (envelope) the same time.

\section{B. Transmission Observation}

This underground channel-wave seismic exploration place is at king 090606 working face Shouyang section Shangxi province (see Fig.3), the ground elevation $1117 \sim 1139 \mathrm{~m}$, working Face elevation of $860 \sim 910 \mathrm{~m}$, depth of $180 \mathrm{~m}$. The working face strike length $714 \mathrm{~m}$, mining width $185 \mathrm{~m}$. Carboniferous Taiyuan Coal Group 9\# coal, coal is lean coal. Roadway expose coal thickness $4.1 \sim 4.3 \mathrm{~m}$, average $4.2 \mathrm{~m}$, coal seam stability, simple structure. Seam dip $7 \sim 15$, the average 11. According 090,604 mining face reveals with $8 \#$ Coal Seam spacing of about $3 \sim 5 \mathrm{~m}, 8$ \# coal seam thickness is $0.2 \mathrm{~m}$. Seam Roof is siltstone rock layer, horizontal distance

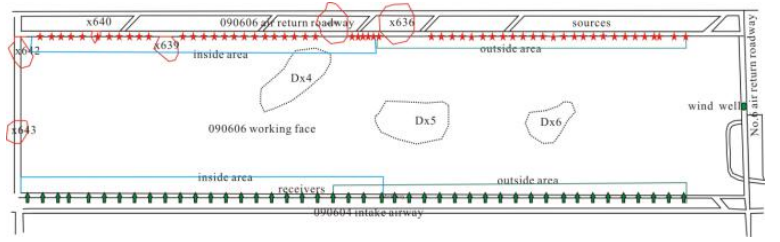

Fig.3 Channel wave seismic exploration work layout diagram in 090606 working face, the red stars indicate source positions and the green arrows are receiver positions.

thickness $3.69 \mathrm{~m}$, medium-grained sandstone false roof, thickness $0.05 \mathrm{~m}$. Direct floor siltstone, average thickness $1.60 \mathrm{~m}$, pseudo bottom of mudstone, the average thickness of $0.10 \mathrm{~m}$. Mine Collapse Column regional development density of $43 / \mathrm{km} 2$. Roadway has revealed subsided column 6 .

Based on the detection target and roadway environmental conditions, gun point arranged this channel wave exploration along the 090,606 return air alley, wave detection point arranged along the 090,606 alley belts, measure working face length $650 \mathrm{~m}$, points inside and outside two parts of observation, which in segments shot points 27 , artillery pitch $10 \mathrm{~m}, 24$ detection point receives, detection pitch $15 \mathrm{~m}$; outer segments shot points 29 , artillery pitch $10 \mathrm{~m}, 24$ detection point receives, detection point interval $15 \mathrm{~m}$.

\section{Processing and Result}

From the original seismic record, in turn the direct $\mathrm{P}$ wave, $\mathrm{S}$-wave and the channel-wave signal reached, $\mathrm{P}$ wave and groove wave signal is clear, easy to identify, S-wave signal is not obvious, demonstrated in Fig.4, the figure outside the paragraph 10 shot records, not found scattering channel wave caused by collapse column in the original seismic record.

By narrowband filtering the received signal obtained channel frequency wave dispersion curve, rays pass through the normal area , channel wave dispersion curves smooth and continuity, Eric phase group velocity at $1000 \pm 200 \mathrm{~m} / \mathrm{s}$, Eric phase frequency $150 \pm 50 \mathrm{~Hz}$. Fig. 5 single dispersion curves for the 10th excited and received point, dispersion curve from 50$350 \mathrm{~Hz}$ good continuity $(50-350 \mathrm{~Hz}$ band-pass filter with the original data filtering), in the $50-125 \mathrm{~Hz}$ range, the channel wave group velocity varies from $4000 \mathrm{~m} / \mathrm{s}$ to $1100 \mathrm{~m} / \mathrm{s}$, the channel wave group velocity increase down with frequency rapidly up, in $125-250 \mathrm{~Hz}$ range, channel wave group velocity varies slowly with frequency change, at $>250 \mathrm{~Hz}$ range, the group velocity of the wave is stable at $800 \mathrm{~m} / \mathrm{s}$.

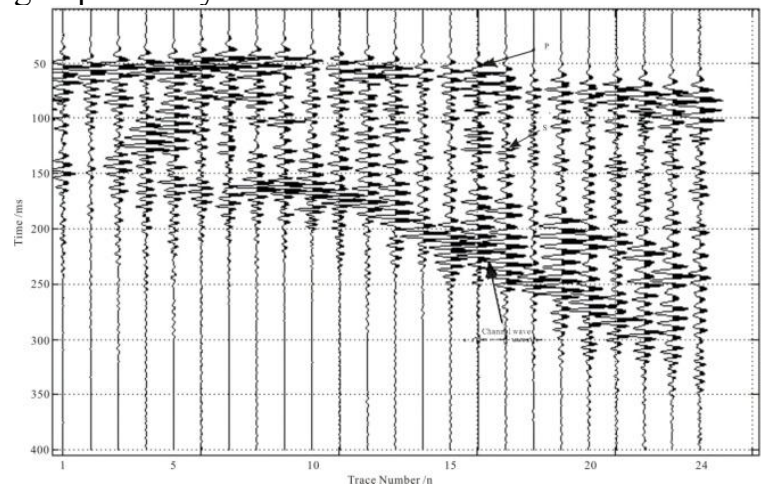

Fig.4 Outside area No.10 shot gathers

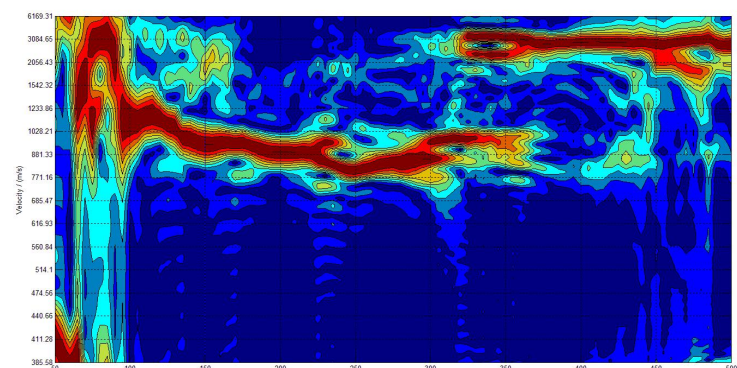

Fig.5 Outside No.10 shot and No.10 receiver dispersion curve, 5-350Hz channel wave frequency range, Airy phase frequency $150 \mathrm{~Hz}$ and group velocity about $1000 \mathrm{~m} / \mathrm{s}$

On the dispersion curves picked up 1344 Eric phase travel time, by inversion calculation of speed and absorption coefficient tomographic to obtain the velocity profile (Fig. 6) and the relative attenuation coefficient distribution (Fig.7). As from the slot wave exploration results figure, at working face return airway and cut expose collapse column X636, X638, $\mathrm{X} 643$ position velocity changes significant, the velocity is relatively high, greater than $1200 \mathrm{~m} / \mathrm{s}$, relative attenuation coefficient greater than 0.3 . Many strong absorption abnormal areas found bury many obvious speed at the working face, according to the imaging results divided into the 10 abnormal areas, demonstrated in Figure 6

Divided 1-9 anomalous bodies are ranging sizes and shapes of collapse column, which has been revealed collapse column $1,6,7$ by roadway, the 1 st Face collapse column toward to working face extends within approximately 40m; the 6 th collapse column extends into Face approximately 40m; the 7th collapse column extends into the working face approximately $20 \mathrm{~m}$; the 2 nd subsided column major axis $30 \mathrm{~m}$, minor $10 \mathrm{~m}$; the 3 rd subsided column long axis about $55 \mathrm{~m}$, a minor axis of about $30 \mathrm{~m}$; the 4 th Collapse Column the long axis of about 50 
$\mathrm{m}$, a minor axis about $30 \mathrm{~m}$, with the ground 3D seismic survey revealed similar collapse column DX4 location; the 8th collapse column long axis approximately $20 \mathrm{~m}$, minor $10 \mathrm{~m}$; the 9th collapse column long axis approximately $50 \mathrm{~m}$, minor axis about $20 \mathrm{~m}$; at the working face in the horizontal 410 $510 \mathrm{~m}$, ordinate $60-100 \mathrm{~m}$ regional area distribute multiple anomalies of diameter less than $10 \mathrm{~m}$; the DX5 and DX6 collapse column that 3-D seismic exploration showed but revealed normal from the tomography results, No 2. -5 collapse column exist, seriously affect the safety of mining coal. Number 1-9 collapse column are verified by dropping, position deviation is less $10 \mathrm{~m}$. dropping not verify the 10 th anomalies, reason analyzed : the border affects and less data near the border, due to impact through the 8th abnormalities and the other multiple diameter is less than $10 \mathrm{~m}$, and by the impact of irregular roadway wave reflection wave nearby.

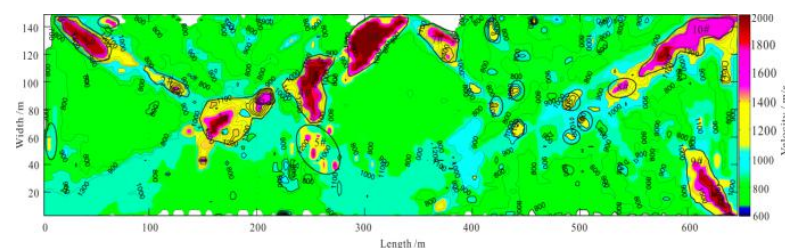

Fig.6 090606 working face channel wave group velocity distribution, black circles are abnormal area of high velocity.

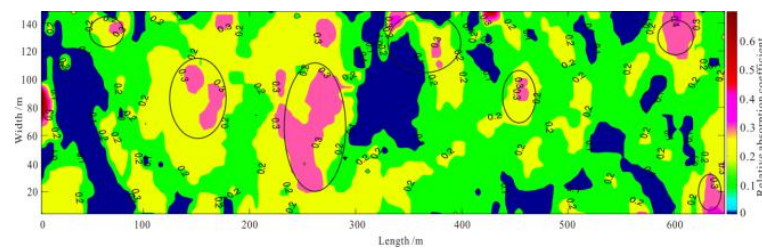

Fig.7 090606 working face channel wave relative attenuation coefficient distribution, black circles are abnormal area of strong absorption.

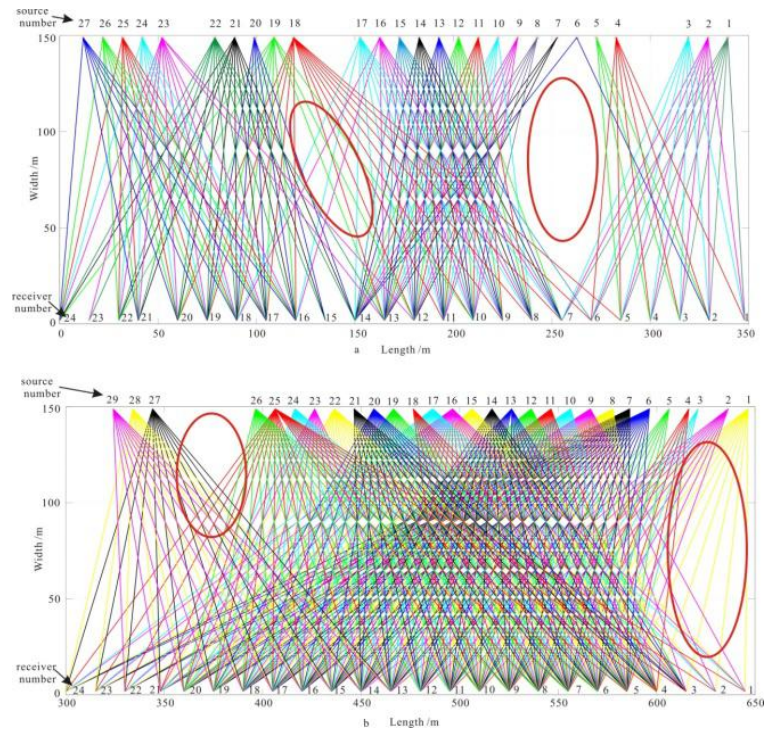

Fig.8 090606 working face channel wave ray coverage diagram, a inside area observation, $b$ outside area observation, red circles are abnormal area

According continuity of channel wave frequency dispersion curve, the ray path projected into the working face that frequency dispersion curve continuity is complete, Fig. 8, elucidate the high probability of anomalies presence in the ray path sparse area, red circled region in Fig.8, consistent with the tomography results in 3-6th and the 9th abnormal area.

\section{CONCLUSIONS}

Through channel wave field characteristics and frequency dispersion analysis, and tomography, obtained detailed distribution diagram of column morphology, get the following understanding:

- Channel transmission tomography method is an effective method to explore seam subsidence column, detection results verified by mine stopping.

- In contrast, channel transmission method has the more high precision than the 3D-seismic exploration, exclude the DX5, DX6 abnormal area delineated by threedimensional seismic exploration, and the position, shape of the 4th abnormal area delineated by channel transmission exploration is more accurate than that of $3 \mathrm{D}$ seismic exploration.

- In this experiment, subsided column anomalies densely distributed, shapes difference and plane geometry area be small, increasing the difficulty of lateral resolution; Collapse Column irregular borders, forward scattering is weak, transmission wave energy weak; Collapse Column fillings the main pieces of roof coal seam collapse pillars; Physical properties difference between the collapse column and coal seam is relatively small, so the wave field of collapse column anomalous bodies is not easy to identify.

\section{REFERENCES}

[1] ZHANG Mao-lin, YIN Shang-xian, 2007, Forming process of subsided column in coalfields of North China:Coal Geology \& Exploration,35,26 29.

[2] YIN Shang-xian,WU Qiang, WANG Shang-xu, 2004,Studies on characters and forming mechanism of karstic collapse columns at mine area of North China: Chinese Journal of Rock Mechanics and Engineering, 23,120 123.

[3] CAO Zhi-yong, WANG Wei, YANG De-yi, LI Fei, 2008,Coalfield Subsided Column Wave field Modeling and Analysis. Journal of Taiyuan University of Technology, ,39, 841 843.

[4] WU Shou-hua, ZHOU Guo-xing, YANG Su-xia, HE Huangsheng,2004,Analysis for the seismic response characteristics of collapse column. Coal Geology \& Exploration, 32,52 54.

[5] MENG Xian-min, JIANG Wei-ping, 2009, Elastic Impedance Inversion Technology and Its Application Effect: COAL GEOLOGY OF CHINA, 21,52 54

[6] YANG X D, 2008,Applied Results of 3D Seismic Prospecting in Shallow Coal Seam Bur ied Areas: Coal Geology of China, 20,62 64.

[7] LIU J G, ZHAO Q B, YIN S X,2011, Coalfield hidden karst collapse column probing and comprehensive treatment technology: China Coal Industry Publishing House.

[8] Marshall Miller Associates,2006,An analysis of in-seam seismic reflection techniques to identify and locate abandoned underground mine voids in advance of mining :Prepared for the U.S. Department of Labor Mine Safety and Health Administration.

[9] S.A. Greenhalgh, B. Zhou, D.R. Pant and A. Green, 2007, Numerical study of seismic scattering and waveguide excitation in faulted coal seams: Geophysical Prospecting, 55,185-198.

Ji G Z, Cheng J Y, Zhu P M, 2011, Numerical Simulation of Seam Love Type Channel-Wave and Analysis on Dispersion Features: Coal Science and Technology , 39, 106-109. 